\title{
Design of segmented thermoelectric Peltier coolers by topology optimization
}

\author{
Lundgaard, Christian; Sigmund, Ole
}

Published in:

Applied Energy

Link to article, DOI:

10.1016/j.apenergy.2019.01.247

Publication date:

2019

Document Version

Peer reviewed version

Link back to DTU Orbit

Citation (APA):

Lundgaard, C., \& Sigmund, O. (2019). Design of segmented thermoelectric Peltier coolers by topology optimization. Applied Energy, 239, 1003-1013. https://doi.org/10.1016/j.apenergy.2019.01.247

\section{General rights}

Copyright and moral rights for the publications made accessible in the public portal are retained by the authors and/or other copyright owners and it is a condition of accessing publications that users recognise and abide by the legal requirements associated with these rights.

- Users may download and print one copy of any publication from the public portal for the purpose of private study or research.

- You may not further distribute the material or use it for any profit-making activity or commercial gain

- You may freely distribute the URL identifying the publication in the public portal

If you believe that this document breaches copyright please contact us providing details, and we will remove access to the work immediately and investigate your claim. 


\title{
Design of segmented thermoelectric Peltier coolers by topology optimization
}

\author{
Christian Lundgaard and Ole Sigmund \\ Department of Mechanical Engineering Techinical University of Denmark, Nils Koppels Allé 404, DK-2800 Kgs. Lyngby, Denmark
}

\begin{abstract}
A density-based topology optimization approach is used to optimize the cooling power and efficiency (coefficient of performance) of thermoelectric coolers by spatially distributing two different thermoelectric materials in a two dimensional design space. With basis in three numerical examples we identify important model parameters, such as the choice of objective function, the temperatures of the thermal reservoirs, the heat transfer rates and the available electrical energy. By using the topology optimization approach, we demonstrate that the cooling power and efficiency of thermoelectric coolers can be improved by $48.7 \%$ and $11.4 \%$, respectively, compared to optimization results from in the literature.
\end{abstract}

Keywords: Topology optimization, thermoelectric energy conversion, Peltier coolers, segmentation

\section{Introduction}

Thermoelectricity is a physical phenomenon which concerns the interaction between electric and thermal energy in semi-conducting materials. Thermoelectricity can be characterized by two separately identified effects, the Seebeck effect which concerns the conversion of thermal energy into electric energy, and the Peltier effect which concerns the conversion of electric energy into thermal energy [1]. With reference to the sketch in Fig. 1, a thermoelectric cooler is a solid-state heat pump which uses the Peltier effect to convert electrical energy into a thermal energy flux and hereby providing cooling power at a specified surface.

Compared to vapor-compression refrigeration systems, thermoelectric coolers offer reliable and silent operation due to the simple system designs without moving parts and circulating fluids. Due to the miniature scales of the systems and their flexibility in packaging and integration, thermoelectric coolers are often seen in applications, such as cooling of electronics [2]. As thermoelectric coolers are fabricated and operate without using chlorofluorocarbons or other chemicals that may be harmful to the environment, thermoelectric coolers are often considered as an environmentally friendly alternative to conventional refrigeration systems. Thermoelectric coolers are therefore by many researchers in science and industry often predicted to be an important entrant in the green energy changeover [3].

A thermoelectric cooler consists of a number of $\mathrm{mod}$ - ules which are connected electrically in series and thermally in parallel, see Fig. 1. The modules are build up by three main components: conductors, legs and substrates. The conductors connect the legs electrically and the substrates constitute the interface between the ambient and the compartment. Thermoelectric coolers can be enhanced by so-called segmentation where two dissimilar thermoelectric materials are connected thermally and electrically in simple one dimensional interfaces. However, it is still an open question if the segmentation approaches can be improved further by allowing two and three dimensional geometries of the segmented materials. In the present study we seek to answer this question by using a density-based topology optimization approach to spatially distribute two different thermoelectric materials in a thermoelectric leg and hereby optimize the cooling power and efficiency of thermoelectric coolers. The paper is therefore concerned with energy conversion, optimal use of energy resources and analysis and optimization of energy processes.

Compared to vapor-compression refrigeration systems, thermoelectric coolers are so far limited to niche applications due to their relatively low operational cooling power and efficiency (coefficient of performance). Despite a considerable amount of scientific efforts, performance improvements of thermoelectric coolers are still required to increase the range of applications [4, 3]. The main efforts to increase the performance of thermoelectric coolers have so far been a broad search for identification and development of advanced thermoelec- 


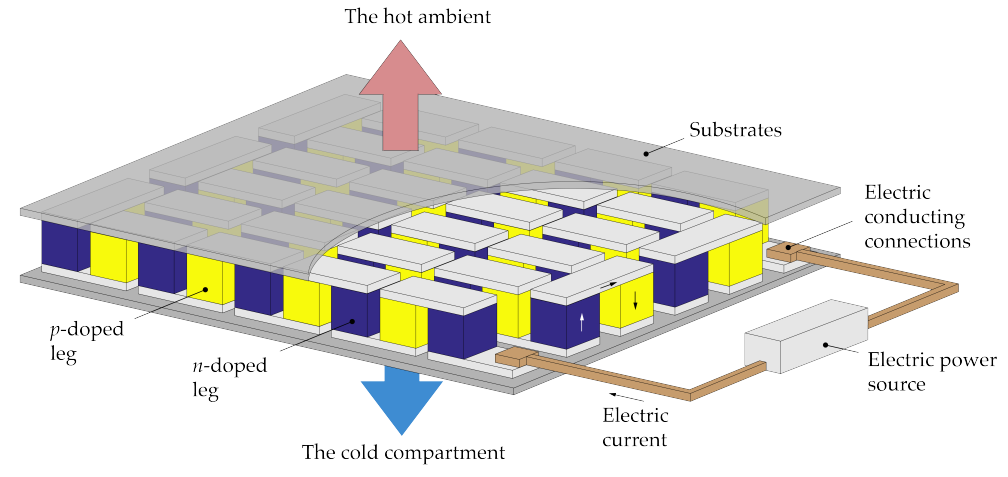

(a) Thermoelectric cooling device

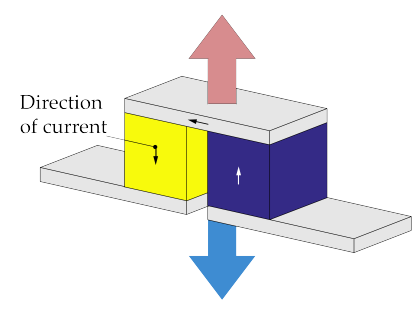

(b) Thermoelectric module

Figure 1: A schematic of a thermoelectric coolor and thermoelectric module

tric materials $[5,6]$, however, in this paper we address a purely mathematical optimization approach aiming at finding the best spatial distributions of available materials in order to optimize a specified performance measure.

In the literature, the performance of thermoelectric coolers has been characterized in different ways, see e.g. Seifert, Müller, and Walczak [7] or Bian, Wang, Zhou, and Shakouri [8]. As we see it, the performance measures can be divided into four categories: (A) the temperature at the compartment surface, $f_{T}$. (B) the heat flux at the compartment surface, $f_{Q}$. (C) the coefficient of performance at the compartment surface, $f_{\mu}$. (D) and the dimensionless figure-of-merit of the device, $f_{\mathrm{ZT}}=$ $\alpha \sigma / \kappa$, where $\alpha$ is the Seebeck coefficient, $\sigma$ is the electric conductivity, $\kappa$ is the thermal conductivity and $T$ is the temperature. In this study we address objectives (A), (B) and $(\mathrm{C})$.

Only a minor part of the scientific efforts concerned with improving thermoelectric energy conversion takes basis in mathematical optimization approaches. The available approaches can generally be sorted in three categories: (a) functionally graded material studies, (b) compatibility and segmentation approaches, and (c) geometrical optimization approaches. The topology optimization approach proposed in present thesis is a subclass of (b).

Functionally graded material studies are aiming at identifying spatial profiles of relevant material parameters which optimize a prescribed performance measure of thermoelectric coolers and generators. The design solutions of functionally graded material studies are characterized by macroscopic gradients in the material parameters, which may be linked to the composition (including doping) or micro structures of the functional properties of the material [9].

In the works of Müller, Walczak, and Seifert [10],
Bian and Shakouri [11], Bian and Shakouri [12] and Bian, Wang, Zhou, and Shakouri [8], the coefficients in arbitrary interpolation functions for the spatial profiles of Seebeck coefficient, $\alpha(x)$, and the electric conductivity, $\sigma(x)$, were optimized with basis in parameter studies and non-gradient algorithms. Such algorithms are inadequate for design problems with many design variables such as the density-based topology optimization approach [13].

Later, a gradient-based optimization approach for functionally graded materials was introduced in Gerstenmaier and Wachutka [14] and later extended to physically realistic boundary conditions in Gerstenmaier and Wachutka [15]. The topology optimization methodology $[16,17]$ used in this study is also gradient-based and supports the same type of boundary conditions as Gerstenmaier and Wachutka, however, the two methodologies take completely different offset and modeling approaches and hereby result in completely different design solutions.

Compatibility approaches were originally suggested for thermoelectic generators in the work of Ursell and Snyder [20] and have later been developed in a series of studies in e.g. Seifert, Müller, and Walczak [21], Snyder, Toberer, Khanna, and Seifert [22] and Seifert, Pluschke, and Hinsche [23]. By identification of compatible materials, it has been shown that the performance of thermoelectic generators and thermoelectic coolers can be considerably improved by segmentation. Compatible materials operate optimally under the same external electrical resistance and are therefore suited for being segmented, i.e. connected thermally and electrically in series. The design solutions of the compatibility approach are generally characterized by one dimensional (1D) line interfaces between the materials phases, where the design solutions of the topology optimization approach support arbitrary two dimensional (2D) features. 
The compatibility approach is, as the functionally graded material approach, related to the topology optimization methodology, however the approaches take very different offsets and converge to different design solutions.

By studying the volume fraction between two materials connected thermally and electrically in series, Yang, Xie, Ma, and Lei [24] presented a mathematical optimization approach aiming at increasing the effective figure-ofmerit of two segmented materials. It was shown that the figure-of-merit of the composite medium could exceed the figure-of-merit of the constitutive materials, if the electric potential difference was chosen sufficiently large when evaluating the electric conductivity. A related approach was utilized to optimize the conversion efficiency in Yang, Ma, Lei, and Liu [25].

System configurations where a vertically directed heat flux is converted into a horizontally directed electric current are often reffered to as off-diagonal problems. These problems were addressed in Sakai, Kanno, Takahashi, Tamaki, Kusada, Yamada, and Abe [26], who studied the tilting angle and volume fraction between two segmented materials in order to optimize the device figure-of-merit. The approach, which has been theoretically improved and discussed in ? [27], was limited to fixed temperature boundary conditions, simple topological design solutions and constant material parameters.

In the work of Schilz, Müller, Helmers, Kang, Noda, and Niino [9] and Müller, Walczak, and Seifert [10], the cooling power of thermoelectric coolers was optimized by maximizing the local figure-of-merit with respect to the local temperature conditions of the device during operation. The interaction between figure-of-merit and electric power output for thermoelectric generators was addressed in Lundgaard and Sigmund [27], and we do therefore not consider this measure in the present paper.

The topology optimization approach used in this study uses a completely different offset and modeling approach and converges to different design solutions compared to the functionally graded material, compatibility and homogenization approaches. The topology optimized design solutions are characterized by two separately identified material phases and two dimensional features, and if the design problems are solved for physical material parameters, the design solutions can straight-forwardly be interpreted and manufactured without any consideration of the local functional properties of the materials.

\section{The design problem}

The topology optimization methodology $[16,17]$ used in this study is based on a finite element formulation of the generalized Ohm's and Fourier's law [28], the
Table 1: List of important variables used throughout the study.

\begin{tabular}{|c|c|}
\hline Variable & Description \\
\hline$\Gamma^{H}$ & $\begin{array}{l}\text { Boundary at the ambient } \\
\text { (thermal hot reservoir) }\end{array}$ \\
\hline$\Gamma^{C}$ & $\begin{array}{l}\text { Boundary at the compartment } \\
\text { (thermal cold reservoir) }\end{array}$ \\
\hline$T^{H}$ & Temperature of the ambient at $\Gamma^{H}$ \\
\hline$T^{C}$ & Temperature of the compartment at $\Gamma^{C}$ \\
\hline$T^{H C}$ & Abbreviation of $T^{H}$ and $T^{C}$ combined \\
\hline$h^{H}$ & Convection coefficient at $\Gamma^{H}$ \\
\hline$h^{C}$ & Convection coefficient at $\Gamma^{C}$ \\
\hline$h^{H C}$ & Abbreviation of $h^{H}$ and $h^{C}$ combined \\
\hline$\Delta T$ & $\begin{array}{l}\text { Temperature difference } \\
\text { between } \Gamma^{H} \text { and } \Gamma^{C}\end{array}$ \\
\hline$\Delta V$ & $\begin{array}{l}\text { Electric potential difference } \\
\text { between } \Gamma^{H} \text { and } \Gamma^{C}\end{array}$ \\
\hline$\vec{T}$ & The temperature field $[\mathrm{K}]$ \\
\hline$\vec{V}$ & The electric potential field [V] \\
\hline$\vec{Q}_{x}, \vec{Q}_{y}$ & $\begin{array}{l}\text { The thermal heat flux }\left[\mathrm{W} / \mathrm{m}^{2}\right] \\
\text { in } x \text { and } y \text {, respectively }\end{array}$ \\
\hline$\vec{J}_{x}, \vec{J}_{y}$ & $\begin{array}{l}\text { The electric current density } \\
{\left[\mathrm{A} / \mathrm{m}^{2}\right] \text { in } x \text { and } y \text {, respectively }}\end{array}$ \\
\hline$f_{T}$ & Temperature average at $\Gamma^{C}$ \\
\hline$f_{Q}$ & Heat flux at $\Gamma^{C}$ \\
\hline$f_{P}$ & Electric power input at $\Gamma^{C}$ \\
\hline$f_{\mu}$ & $\begin{array}{l}\text { Coefficient of performance, } \\
f_{\mu}=f_{Q} / f_{P} \text {, at } \Gamma^{C}\end{array}$ \\
\hline$\Omega_{D}$ & Design domain \\
\hline$L_{x}$ & Length of $\Omega_{D}$ in $x$ \\
\hline$L_{y}$ & Length of $\Omega_{D}$ in $y$ \\
\hline
\end{tabular}

method of moving asymptotes [29], adjoint sensitivity analysis [30], and various filter operations [31, 32]. The framework supports advanced physical modeling concepts such as temperature dependent material parameters, complex geometries and advanced boundary conditions. A detailed description and implementation details of the framework can be found in Lundgaard and Sigmund [19], however we will briefly discuss the most important features of the framework in the following. In this connection we introduce several variables which we have summarized in Tab. 1.

\subsection{Physical model}

The design problem takes basis in the sketch in Fig. 2, where a single leg of a single module of a single thermo- 


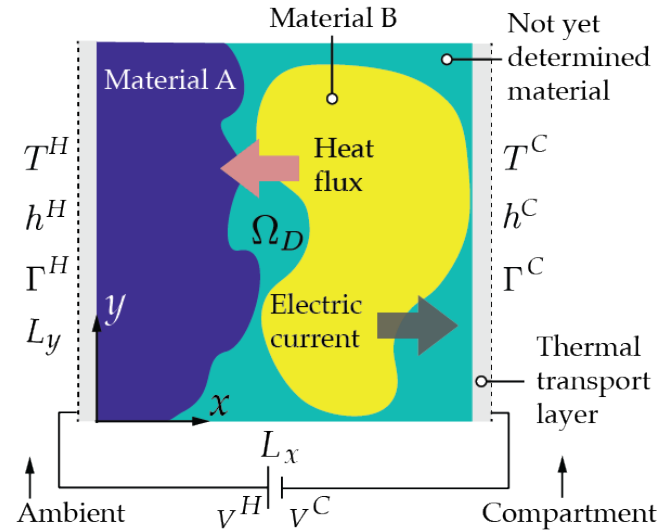

Figure 2: A sketch of the design problem. The aim of the design problem is to spatially distribute two materials, Material A and Material B (see Tab. 2 for material paramters), to maximize the cooling power, $f_{T}$ or $f_{Q}$, or the efficiency (coefficient of performance), $f_{\mu}$, in the compartment.

electric cooler is considered. The boundary conditions and the model parameters are inspired by a household refrigerator being powered by thermoelectric cooling. The hot ambient with temperature, $T^{H}$, and the cold compartment, $T^{C}$ are separated by the leg with dimensions $\left\{L_{x}, L_{y}\right\}=\{0.005,0.005\}[\mathrm{m}]$.

Material A and B (see Fig. 2) are assumed temperature independent, similar to the material parameters used in the analytic derivations in the work of [24], and listed in Tab. 2. Since the present work concerns the development of a general numerical design approach applicable to any types and choices of materials, we do not include experimental verifications. However, for experimental validation of Material A and B considered in this study, we refer to the work of Yang, Xie, Ma, and Lei [24].

We also decided to limit the design problems to linear materials, as the design features of the counterpart design solutions with non-linear materials are more challenging to interpret. However, the framework is easily extended to support temperature dependent materials, see Lundgaard and Sigmund [19].

\subsubsection{Equations}

The basic partial differential equations of thermoelectricity are constrained by Fourier's and Ohm's generalized law. The continuity of thermal energy and electric charge are in $\Omega_{D}$, see Fig. 2, given by [1]:

$$
\begin{array}{lll}
\nabla \cdot \vec{Q}=\dot{q} & \text { in } & \Omega_{D} \\
\nabla \cdot \vec{J}=0 & \text { in } & \Omega_{D}
\end{array}
$$

where $\nabla$ denotes the spatial derivative with respect to Cartesian directions $x$ and $y ; \vec{Q}=\left\{Q_{x}, Q_{y}\right\}$ is the heat flow density in $x$ and $y\left[\mathrm{~W} / \mathrm{m}^{2}\right] ; \dot{q}=\vec{J} \cdot \vec{E}$ is the Joule heating term $\left[\mathrm{W} / \mathrm{m}^{3}\right] ; \vec{E}=-\nabla V$ is the electric field $[\mathrm{V} / \mathrm{m}]$; $V$ is the electric potential and $\vec{J}=\left\{J_{x}, J_{y}\right\}$ is the electric current density in $x$ and $y\left[\mathrm{~A} / \mathrm{m}^{2}\right]$. In thermoelectric analysis, the thermal and electric energies are coupled by the constitutive equations:

$$
\begin{aligned}
& \vec{Q}=T \alpha \cdot \vec{J}-\kappa \cdot \nabla T \\
& \vec{J}=\sigma \cdot(\vec{E}-\alpha \cdot \nabla T)
\end{aligned}
$$

where $T$ is the temperature $[\mathrm{K}]$.

\subsubsection{Newton's law of cooling}

With reference to the simple rectangular geometry of the thermoelectric cooler in Fig. 2 and the relatively small length scales of the modules, we assume that the heat transfer between the module, the ambient and the compartment can be modeled by Newton's law of cooling. Using Newton's law of cooling, it is assumed that the thermal heat transfer rate at $\Gamma^{H}$ and $\Gamma^{C}$ is proportional to the temperature difference between the ambient, the compartment and the module:

$$
\vec{n} \cdot \vec{Q}=h^{H}\left(T-T^{H}\right) \quad \vec{n} \cdot \vec{Q}=h^{C}\left(T-T^{C}\right)
$$

where $h^{H}$ and $h^{C}$ denote the heat transfer coefficients $\left[\mathrm{W} / \mathrm{m}^{2} \mathrm{~K}\right]$ on $\Gamma^{H}$ (the ambient) and $\Gamma^{C}$ (the compartment), respectively; $\vec{n}$ is the vector normal to the surface; and $T^{H}$ and $T^{C}$ denote the temperatures of the hot and cold thermal reservoirs $[\mathrm{K}]$ in $\Gamma^{H}$ and $\Gamma^{C}$, respectively. If the heat transfer coefficients on $\Gamma^{H}$ and $\Gamma^{C}$ are equal, they are combined denoted $h^{H C}$ to reduce the extent of the notation.

In Newton's law of cooling, the heat transfer rate between the leg and the thermal reservoirs is governed by the fluid and the flow types at the boundaries. Convection coefficients of various flow types and flow conditions have been listed in Tab. 3 for reference.

\subsection{Finite element model}

The governing equations in Eqs. (1)-(4) are solved numerically by discretizing the equations into finite elements $[33,28,34]$. The topology optimization framework is set up by introducing a design field $0 \leq \rho \leq 1$, such that the material parameters in the governing equations become functions of the design field, i.e. $\alpha=\alpha(\rho)$, $\sigma=\sigma(\rho)$ and $\kappa=\kappa(\rho)$. By introducing the design variable field, it is possible to control whether a finite element represents Material A or Martial B. This functionality can be used to optimize a specific performance measure, i.e. objective function, of the device. 
Table 2: The Seebeck coefficient, $\alpha$; the electric conductivity, $\sigma$; and the thermal conductivity, $\kappa$ for Material A and B used in the design problem sketched in Fig. 2. The material parameters are copied from Yang, Xie, Ma, and Lei [24].

\begin{tabular}{lrrrrr}
\hline & Color in plots & $\alpha[\mathrm{V} / \mathrm{K}]$ & $\sigma[\mathrm{S} / \mathrm{m}]$ & $\kappa[\mathrm{W} /(\mathrm{m} \cdot \mathrm{K})]$ & $Z^{*}[1 / \mathrm{K}]$ \\
\hline Material A & & $200 \cdot 10^{-6}$ & $110 \cdot 10^{3}$ & 1.60 & $2.75 \cdot 10^{-3}$ \\
Material B & & $270 \cdot 10^{-6}$ & $22 \cdot 10^{3}$ & 0.77 & $2.10 \cdot 10^{-3}$ \\
\hline
\end{tabular}

Table 3: List of convection coefficients for various flow types and flow conditions.

\begin{tabular}{llr}
\hline Flow type & Flow condition & $h^{H C}$ \\
\hline Forced convection & Air over a surface & 100 \\
& Air over a cylinder & 200 \\
& Water in a pipe & 3000 \\
\hline \multirow{2}{*}{ Free convection } & Water and liquids & $50-3000$ \\
& Water & $100-1200$ \\
& Air & $10-100$ \\
& Various gasses & $5-37$ \\
\hline
\end{tabular}

\subsection{The optimization problems}

We believe that three objective functions are important in TE cooling applications: The average temperature over the boundary $\Gamma^{C}, f_{T}$; the $x$-directional heat flux through $\Gamma^{C}, f_{Q}$; and the $x$-directional heat flux divided by the $x$-directional electric energy through $\Gamma^{C}, f_{\mu}$. Objective function $f_{\mu}$ is in the literature often denoted Coefficient Of Performance and abbreviated COP.

The average temperature objective function, $f_{T}$ is defined as:

$$
f_{T}=\frac{1}{L_{y}} \int_{\Gamma^{C}} T \mathrm{~d} S
$$

The heat flux objective function, $f_{Q}$, is given by:

$$
f_{Q}=\int_{\Gamma^{C}} Q_{x} \mathrm{~d} S
$$

where the lowercase $x$ denotes that the $x$ directional component of the field is being considered. The COP objective function, $f_{\mu}$, is given by:

$$
f_{\mu}=\frac{f_{Q}}{f_{P}}
$$

where $f_{P}$ is the $x$-directional electric energy (the power consumption of the module) given by:

$$
f_{P}=\frac{1}{L_{y}} \int_{\Gamma^{C}} V \mathrm{~d} S \int_{\Gamma^{C}} J_{x} \mathrm{~d} S
$$

In one dimensional problems in the literature, Eq. (9), is often simply written as $P=V J$.

The optimization framework is powered by discrete adjoint sensitivity analysis which provides gradients of the objective functions, see Michaleris, Tortorelli, and Vidal [30], Bendsøe and Sigmund [17], Lundgaard and Sigmund [19] for more information.

\section{Results}

Five numerical examples are presented to demonstrate that the topology optimization methodology is suitable for optimizing thermoelectric coolers. By identifying and discussing important model parameters such as the objective functions, Sec. 3.1; the temperatures of thermal reservoirs, Sec. 3.2; the electric power supply, Sec. 3.3; the heat transfer rates, 3.4; and the features of the design solutions, Sec. 3.5.

The design solutions presented throughout the paper are dependent on the applied electric potential difference, $\Delta V$, between $\Gamma^{H}$ and $\Gamma^{C}$, and unless otherwise stated all design solutions are solved for the specific magnitude of $\Delta V$ that provides the best performing design solution.

\subsection{The objective function}

In the first numerical example we investigate the relationship between the objective functions and the design solutions. The study takes basis in the design solutions solved for average temperature, $f_{T}$, heat flux, $f_{Q}$, a sequence of different convection coefficients, $h^{H C}$, and thermal reservoir temperatures of $T^{H C}=300$ in Fig. 3 .

With reference to Fig. 3, the design solutions can generally be characterized by three attributes: (A) the volume ratio between the material phases, (B) the length of the spike-shaped transitions between the material phases, and $(\mathrm{C})$ the position of the transition between the material phases. The attributes (A), (B) and (C) are all governed by the convection coefficients and the choice of objective function.

By comparing the design solutions solved for $f_{T}$ and $f_{Q}$, we notice that for a specific set of convection coefficients, the design solutions are almost similar, compare e.g. Figs. $3 \mathrm{c}$ and $3 \mathrm{~h}$. As design problems solved for $f_{T}$ 


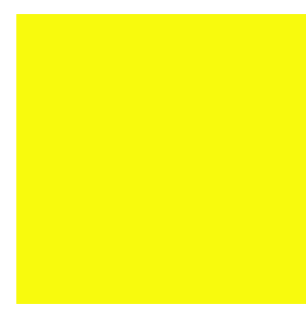

(a) $h^{H C}=175$

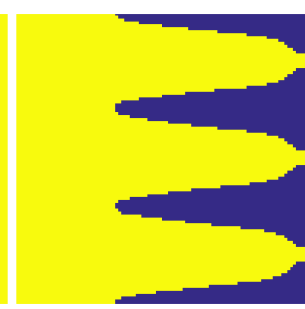

(b) $h^{H C}=276$

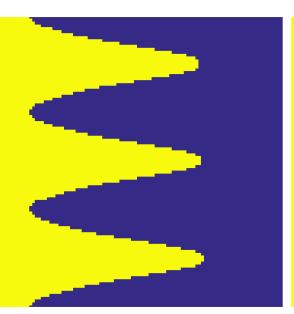

(c) $h^{H C}=412$

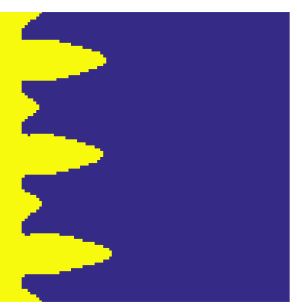

(d) $h^{H C}=530$

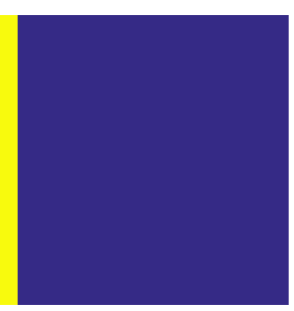

(e) $h^{H C}=1000$

Design solutions solved for $f_{T}$ and $T^{H C}=300$.

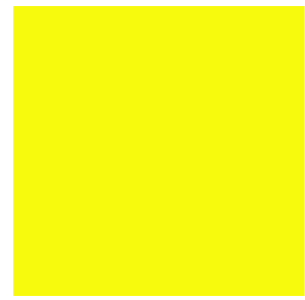

(f) $h^{H C}=175$

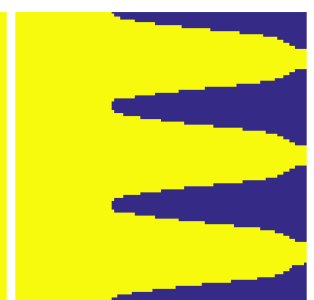

(g) $h^{H C}=276$

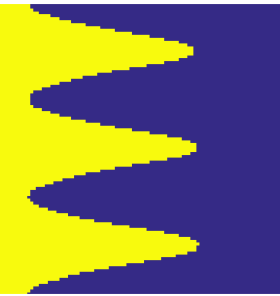

(h) $h^{H C}=412$

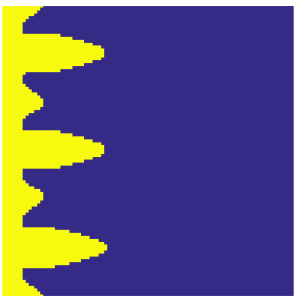

(i) $h^{H C}=530$

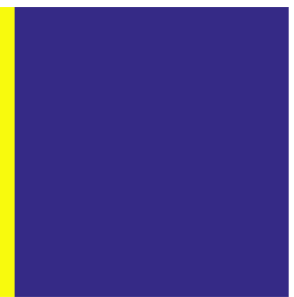

(j) $h^{H C}=1000$

Design solutions solved for $f_{Q}$ and $T^{H C}=300$.

Figure 3: Design solutions solved for the average temperature objective function, $f_{T}$, the heat flux objective function, $f_{Q}$, and various convection coeffcients, $h^{H C}$. The design solutions solved for $f_{T}$ and $f_{Q}$ are almost identical for a specific magnitude of $h^{H C}$ and we hereby conclude that these objectives result in similar design solutions and it is sufficient to consider just one of them.

and $f_{Q}$ result in almost similar design solutions, we will only solve maximum cooling problems for $f_{Q}$ throughout the remaining part of the paper. The equivalence between $f_{T}$ and $f_{Q}$ design problems were also observed in the work by Müller, Walczak, and Seifert [10]. The equivalence between the design problems solved for $f_{T}$ and $f_{Q}$ is after all not surprising, as the temperature difference and heat flux between the same two surfaces basically are the same.

Design solutions solved for $f_{Q}$ and $f_{\mu}$ (coefficient of performance), a sequence of different convection coefficients, $T^{H}=300$ and $T^{C}=260$ have been plotted in Figs. 4. By comparing the designs solutions solved for a specific set of convection coefficients, we observe that the two objective functions result in very different design solutions, compare e.g. $4 \mathrm{~d}$ and $4 \mathrm{i}$.

Three important differences of the design solutions solved for $f_{Q}$ and $f_{\mu}$ are identified: (A) the spike-shaped transitions between the material phases occur for larger convection coefficients in design problems solved for $f_{\mu}$. (B) design solutions solved for $f_{\mu}$ have generally a lower ratio between Material $\mathrm{A}$ and $\mathrm{B}$ which is cost function effective due to the low thermal conductivity of Material $\mathrm{B}$ and $(\mathrm{C})$ the position of the transition between the material phases is different for design problems solved for $f_{Q}$ and $f_{\mu}$.

With basis in these observations, we hereby conclude that the magnitude of the convection coefficients and the objective functions are important model parameters in design problems of thermoelectric coolers.

\subsubsection{Objective function cross-checks}

To verify that the design solutions in Sec. 3.1 indeed have superior performance for the model parameters they were optimized for, we have carried out a crosscheck. Cross-checks are important in many aspects of optimization, as they enlight how much significance we can attribute to the features of the design solutions.

The design solutions in Fig. 3 and 4 are compared with design solutions obtained with the classical segmentation approach. Design solutions solved with the classical segmentation approach are characterized by a one dimensional line interface between the material phases. The main difference between the classical segmented design solutions and the topology optimized design solutions is therefore the two dimensional spike-shaped design features seen in e.g. Fig. 4 i.

In Figs. 5 we have plotted the relationship between $f_{Q}$ and $\Delta V$ for the design solutions solved for $f_{T}, f_{Q}$, $h^{H C}=412$ and $T^{H C}=300$ in Figs. $3 \mathrm{c}$ and $3 \mathrm{~h}$. With reference to the plot, we see that the designs solutions solved with topology optimization outperform the design solutions solved with the classical segmentation approach when $\Delta V$ is tuned such that the highest possible device performances are achieved. Furthermore, we notice that the performance of the design solutions 


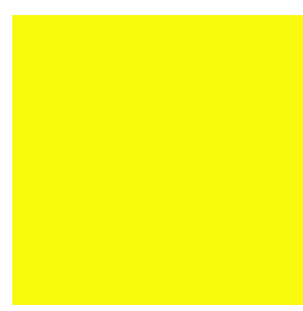

(a) $h^{H C}=191$

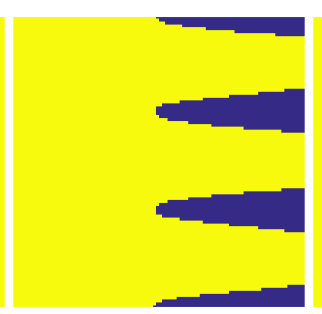

(b) $h^{H C}=530$

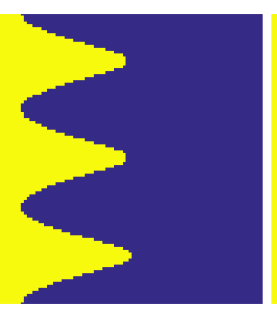

(c) $h^{H C}=1000$

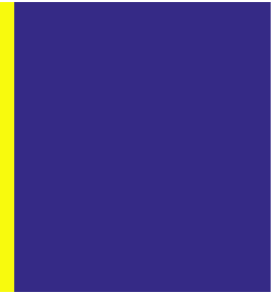

(d) $h^{H C}=2500$ Design solutions solved for $f_{Q}, T^{C}=260$, and $T^{H}=300$

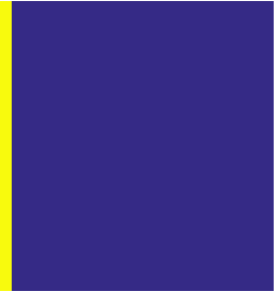

(e) $h^{H C}=3000$

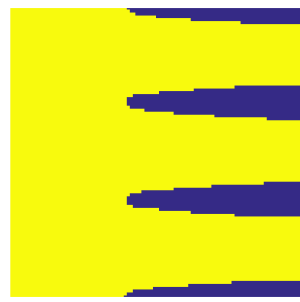

(f) $h^{H C}=191$

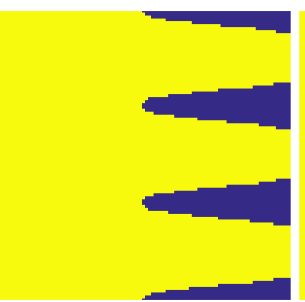

(g) $h^{H C}=530$

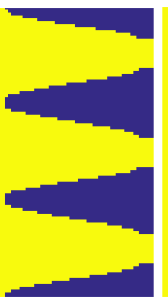

(h) $h^{H C}=1000$

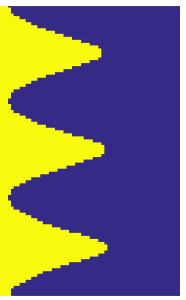

(i) $h^{H C}=2500$

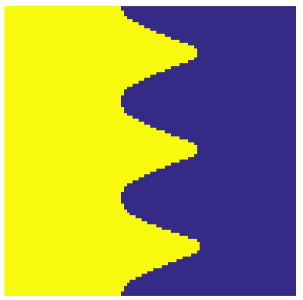

(j) $h^{H C}=3000$

Design solutions solved for $f_{\mu}, T^{C}=260$ and $T^{H}=300$.

Figure 4: Design solutions solved for the heat flux objective function, $f_{Q}$, and the coefficient of performance objective function, $f_{\mu}$, compartment temperature of $T^{C}=260$, ambient temperature of $T^{H}=300$ and various convection coefficients, $h^{H C}$. The design solutions are dependent on $h^{H C}$ and the choice of objective function, for which reason we conclude that these parameters are important in design problems of thermoelectric coolers.

solved for $f_{T}$ and $f_{Q}$ are almost identical which supports what already discussed in Sec. 3.1.

In the sake of completeness, we have cross-checked the design solutions solved for $f_{\mu}, h^{H C}=2500, T^{H C}=$ 260 in Fig. 6. The important features of the cross-check plot are identical to what was already discussed for the cross-check plot in Fig. 5 and we will therefore not discuss this further. However with basis in the crosscheck plots we confidently conclude that (A) we may attribute features to the design solutions, (B) that the objective function is an important model parameter and that $(\mathrm{C})$ the topology optimization approach outperforms the classical segmentation approach.

\subsection{The temperatures of the thermal reservoirs}

The second numerical example is concerned with the relationship between the design solutions and the temperature difference between the ambient and the compartment, $\Delta T$. The study takes basis in the design solutions solved for $f_{Q}, \Delta T=\{0,10,20,30,40\}$, and $h^{H C}=\{191,446,530,700,2500\}$ in Fig. 7.

The design solutions can generally be characterized by two attributes: (A) the ratio between Material A and $\mathrm{B}$ is increased when $T^{C}$ is increased, because an increased $\Delta T$ results in an increased thermal heat transfer rate between the compartment and the ambient. To reduce this heat transfer, the effective thermal conductivity of the design solutions is reduced by increasing the relative amount

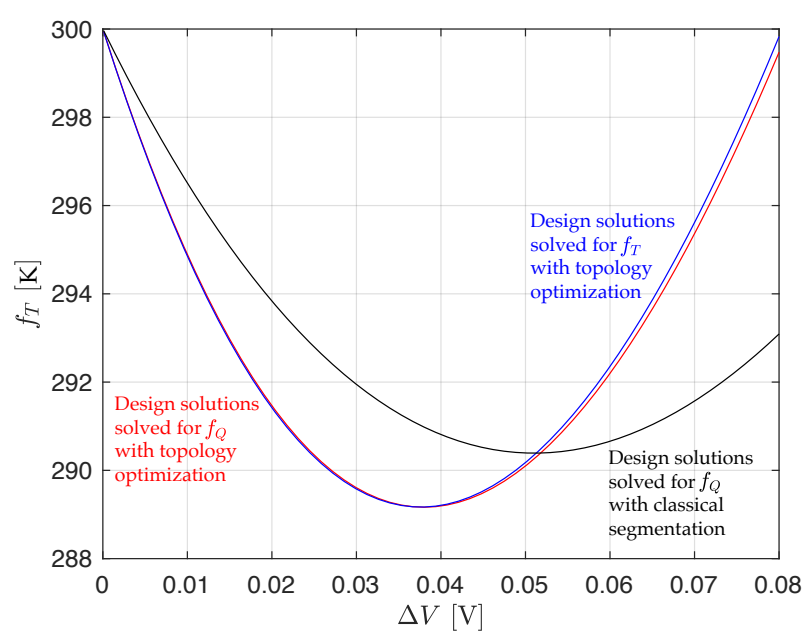

Figure 5: The relationship between the average temperature objective function, $f_{T}$, and the applied electric potential difference, $\Delta V$, for the design solutions solved for $f_{T}, f_{Q}, h^{H C}=412$ and $T^{H C}=300$ in Figs. $3 \mathrm{c}$ and $3 \mathrm{~h}$. The design solution solved with the classical segmentation approach is outperformed by the design solution solved with the topology optimization approach when $\Delta V$ is tuned such that the highest possible device performances are achieved 


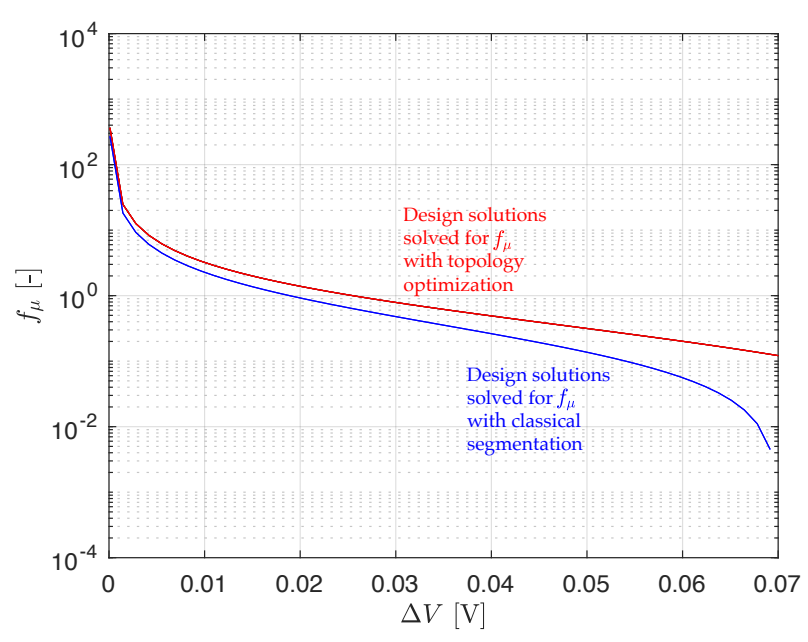

Figure 6: The relationship between the coefficient of performance objective function, $f_{\mu}$, and the applied electric potential difference, $\Delta V$, for the design solutions solved for $f_{Q}, f_{\mu}, h^{H C}=2500, T^{H}=300$ and $T^{C}=260$ in Figs. 4d and 4i. The design solution solved with the classical segmentation approach is outperformed by the design solution solved with the topology optimization approach for all values of $\Delta V$.

of Material A. (B) length and positions of the spikeshaped transitions between the material phases and the increased ratio between Material A and B for increasing $h^{H C}$ is equivalent to what was already discussed in Sec. 3.1 .

As the design solutions are dependent on $h^{H C}$ and $\Delta T$, we conclude that these model parameters are important for design problems of thermoelectric coolers.

\subsubsection{Temperatures of the thermal reservoirs cross- check}

The design solutions solved for $h^{H C}=666$ and $\Delta T=\{0,10,20,30,40\}$ in Fig. $7 \mathrm{~d}, 7 \mathrm{i}, 7 \mathrm{n}, 7 \mathrm{~s}$ and $7 \mathrm{x}$ have been cross-checked with design solutions solved with the classical segmentation approach in Fig. 8. The corresponding cross-check for the design solutions solved for $f_{\mu}, h^{H C}=2500$ and $\Delta T=\{0,10,20,30,40\}$ has been plotted in Fig. 9. Please notice that these designs solutions are not shown in the paper.

With reference to the cross-check plots in Figs. 8 and 9 , we notice that the design solutions solved with topology optimization outperform the design solutions solved with the classical segmentation approach by $48.7 \%$ and $11.4 \%$ with respect to $f_{Q}$ and $f_{\mu}$, respectively. We therefore confidently conclude that topology optimization is suited for optimizing thermoelectric cooling problems.

\subsection{The electric energy supply}

The third numerical example is concerned with the relationship between the design solutions and the electric potential difference between the ambient and the compartment, $\Delta V$. The design solutions solved for $f_{\mu}, h^{H C}=615, T^{C}=280, T^{H}=300$ and $\Delta V=$ $\{0.0169,0.0386,0.0483,0.0579,0.0700\}$ have been plotted in Fig. 10.

The design solutions are obviously dependent on the electric potential difference, and we emphasize the importance of taking this model parameter into consideration in the design problems. The magnitude of $\Delta V$ which result in the best performing design solution is a compromise between the Peltier effect and the Joule heating effect. Design problems solved for too large $\Delta V$ are subject to an objective-ineffective amount of Joule heating, where design problems solved for too low $\Delta V$ are subject to a too low amount of electric energy for powering the Peltier effect. Design solutions solved for inefficiently large $\Delta V$ are also seen in the cross-check plot in Fig. 6 .

The relationships between $\Delta V$ and $f_{Q}$ for the design solutions solved $f_{Q}, T^{C}=280, T^{H}=300$ and $h^{H C}=\{242,327,463,564\}$ have been plotted in Fig. 11. The plot demonstrates that the cooling power and the electric potential difference are coupled with the convection coefficient. We emphasize three important features of the plot: (A) the maximum cooling power is increased as $\Delta V$ is increased. This continues until a specific threshold where the Joule heating effect becomes too dominating and the maximum cooling power begins to decrease. (B) the electrical potential difference necessary to obtain the maximum cooling power is increased as the convection coefficients are increased. $(\mathrm{C})$ the heat flux on $\Gamma^{C}$ is positive for small electric potential differences. Due to the temperature difference between $\Gamma^{C}$ and $\Gamma^{H}$ and the small electric potential difference, the Seebeck effect dominates over the Peltier effect and the thermoelectric cooler is actually working as a thermoelectric generator for these model parameters.

\subsection{The convection coefficient}

The fourth numerical example is a cross-check study which concerns the relationship between the convection coefficients and the design solutions. The study takes basis in Fig. 12, where the relationships between $f_{Q}$ and $h^{H C}$ for the design solutions solved for $f_{Q}, T^{C}=280$, $T^{H}=300$ and $h^{H C}=\{175,276,412,530,1000\}$ have been plotted. The performance of the design solutions has been computed for $h^{H C} \in[0 ; 1000]$ to demonstrate that the design solutions have superior performance for the convection coefficients at which they were optimized. 


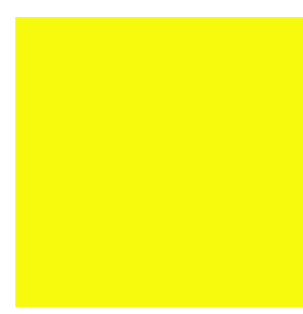

(a) $h^{H C}=293$

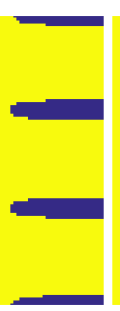

(b) $h^{H C}=412$

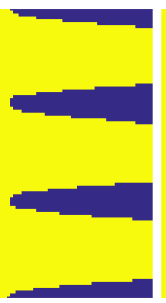

(c) $h^{H C}=497$

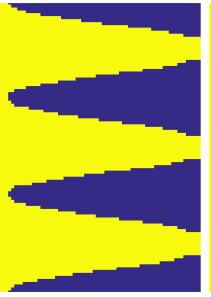

(d) $h^{H C}=666$ Design solutions solved for $T^{C}=260$ and $T^{H}=300$

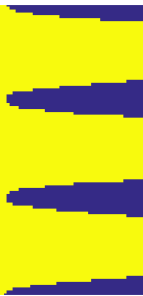

(g) $h^{H C}=412$

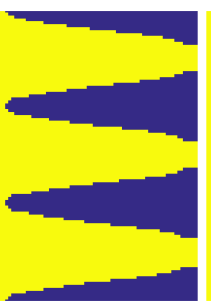

(h) $h^{H C}=497$

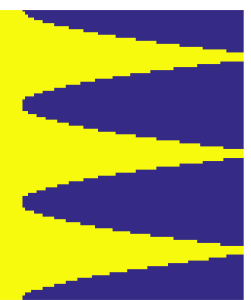

(i) $h^{H C}=666$

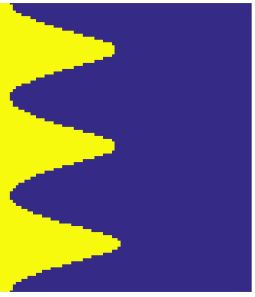

(e) $h^{H C}=1000$

(f) $h^{H C}=293$

Design solutions solved for $T^{C}=270$ and $T^{H}=300$.

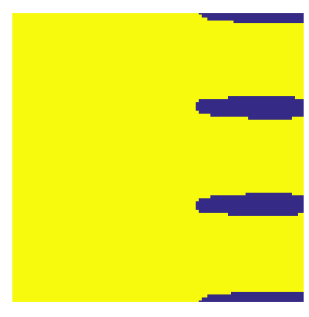

(k) $h^{H C}=293$

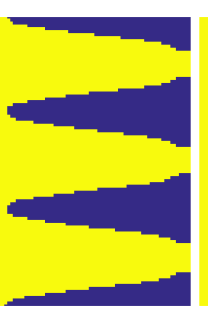

(1) $h^{H C}=412$

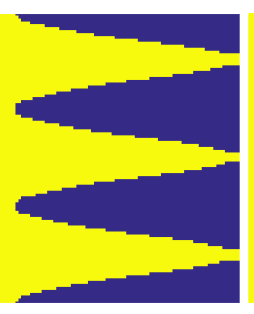

(m) $h^{H C}=497$

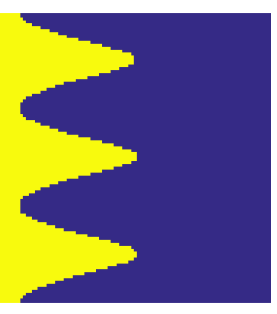

(n) $h^{H C}=666$

$T^{H}=300$.

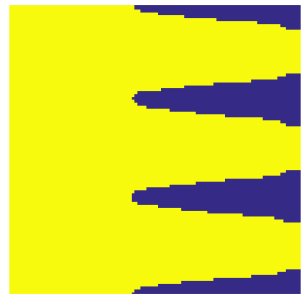

(p) $h^{H C}=293$

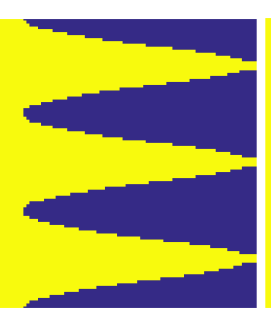

(q) $h^{H C}=412$

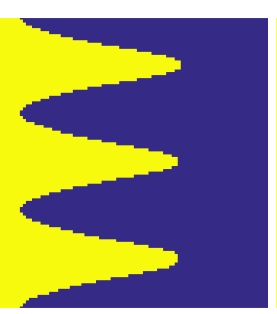

(r) $h^{H C}=497$

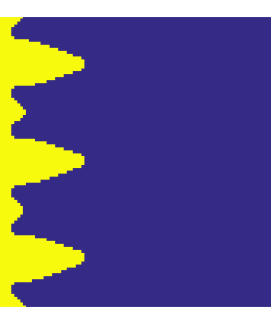

(s) $h^{H C}=666$

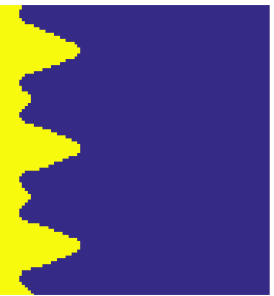

(j) $h^{H C}=1000$

Design solutions solved for $T^{C}=290$ and $T^{H}=300$.

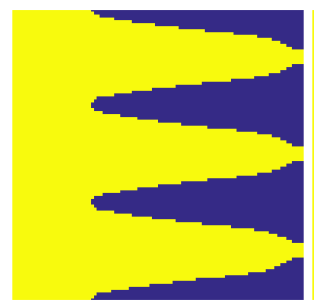

(u) $h^{H C}=293$

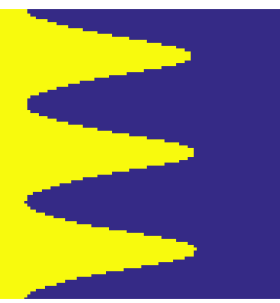

(v) $h^{H C}=412$

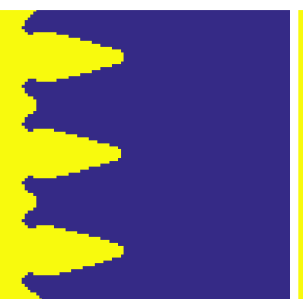

(w) $h^{H C}=497$

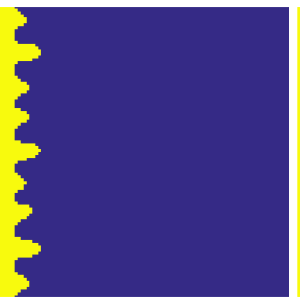

(x) $h^{H C}=666$

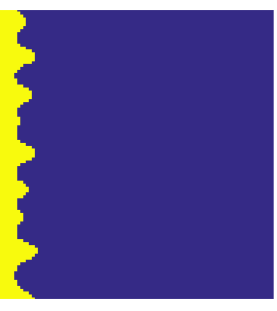

(o) $h^{H C}=1000$

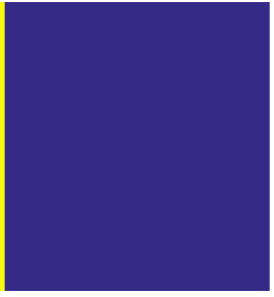

(t) $h^{H C}=1000$

Design solutions solved for $T^{C}=300$ and $T^{H}=300$.

Figure 7: Design solutions solved for the temperature objective function, $f_{T}$, various compartment convection coefficients, $h^{C}$, and various compartment temperatures, $T^{C}$. Due to the dependency between the $h^{H C}$ and $T^{C}$ and the design solutions. we conclude that these model parameters indeed are important for design problems of thermoelectric coolers. 


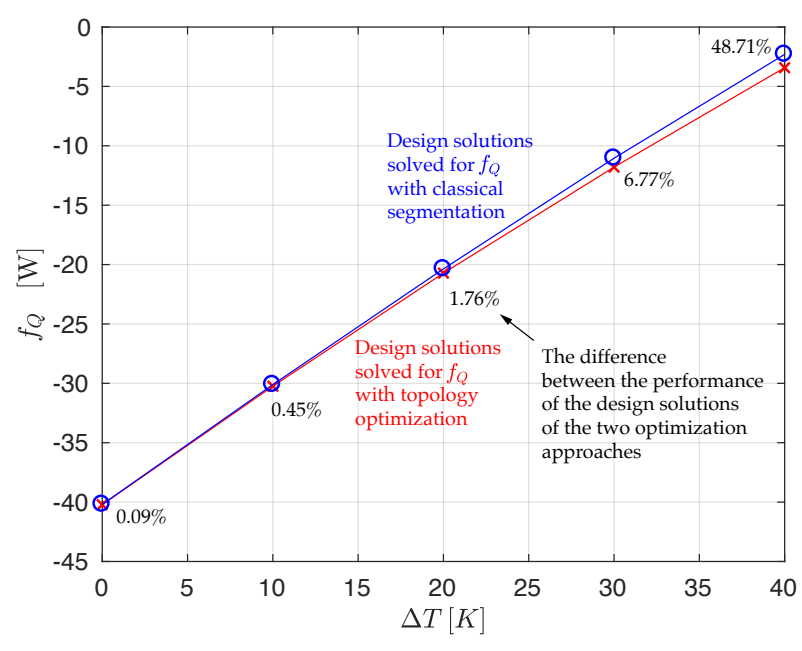

Figure 8: The relationship between the temperature difference between the ambient and the compartment, $\Delta T$, and the heat flux, $f_{Q}$, for the design solutions solved for $f_{Q}$ and $h^{H C}=666$ in Figs. 7d, 7i, 7n, $7 \mathrm{~s}$ and $7 \mathrm{x}$. The design solutions solved with topology optimization outperform the design solutions solved with the classical segmentation approach with up to $48.71 \%$ and the highest heat fluxes are obtained for $\Delta T \rightarrow 0$

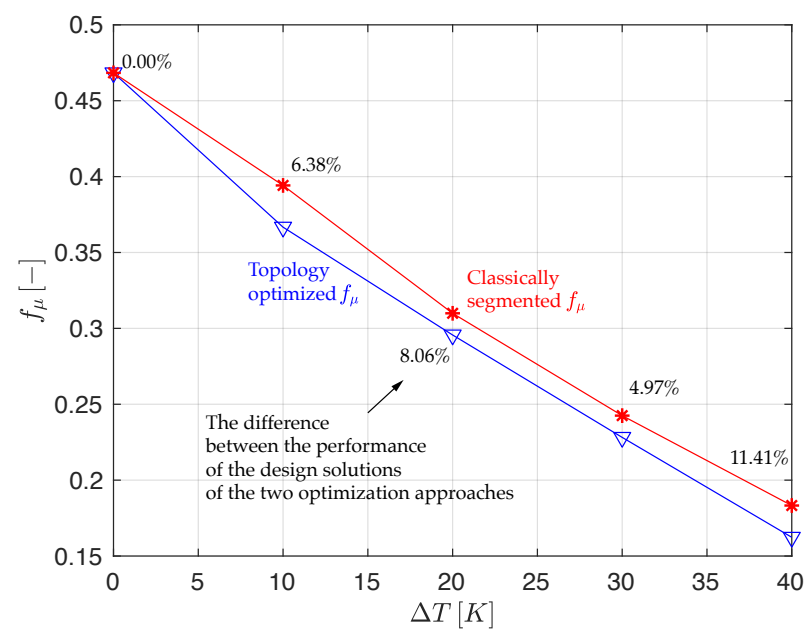

Figure 9: The relationship between the temperature difference between the ambient and the compartment, $\Delta T$, and the heat flux, $f_{Q}$, for the design solutions solved for $f_{Q}$ and $h^{H C}=3000$ (the design solutions are not shown in the paper). The design solutions solved with topology optimization outperform the design solutions solved with the classical segmentation approach with up to $11.4 \%$.
As guidance to understand the plot, we point the attention to the teal/cyan line which illustrates the relationship between $h^{H C}$ and $f_{Q}$ for the design solution solved for $h^{H C}=1000$. As the design solutions are evaluated for $h^{H C}=1000$ we notice that the design solutions solved for $h^{H C}=1000$ outperform the design solutions solved for other convection coefficients.

The same tendency is evident for all the design solutions in Fig. 12, and we thereby conclude that the convection coefficient is an important model parameter and that the topology optimization approach is suited for taking this model parameter into account.

\subsection{Design features}

Throughout the numerical examples presented in Secs. 3.1-3.4, we have seen that some design solutions are characterized by spike-shaped transitions between the material phases. The spike-shaped design features enable the design solutions to operate locally in an intermediate state between the material phases and are therefore "optimal" for some model parameters. The spike-shape design features are indeed a key feature of the design solutions and in this section we suggest a methodology to decompose the $2 \mathrm{D}$ design problems presented throughout Sec. 3.1-3.4 into a 1D design problem which may be solved with an analytical approach.

The state field plots for the design solution in Fig. $7 \mathrm{~m}$ have been plotted in Fig. 13. We notice that despite the 2D design features of the design solution, the $y$-directional gradients in the temperature and electric potential fields are relatively small. Due to the small $y$-directional effects of the design solution, we argue that the $2 \mathrm{D}$ design solution can be approximated by a $1 \mathrm{D}$ design solution where the ratio between the material phase, $v$, can be varied locally as function of $x$. The approximation is therefore achieved by defining a local $x$ directional volume ratio between the materials, $v=v(x)$.

With references to the sketches in Fig. 14, the decomposition is initiated with basis in the finite layered $2 D$ design in Fig. 14a. This design is then decomposed into an "infinite" layered 2D design in Fig. 14b, and finally decomposed into an infinite layered $1 D$ design in Fig. 14c. The infinite in 2D layered designs are here in quotation marks as $h \rightarrow 0$ and $h>0$.

By considering the intermediate design variables in Fig. $14 \mathrm{c}$ as horizontal channels where the $x$-directional volume ratio between the materials is determined by the function $v(x)$, the material parameters can be interpolated 


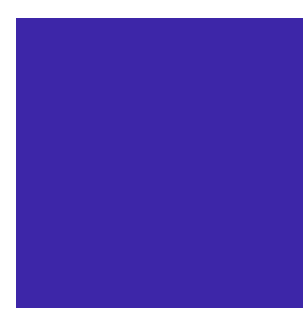

(a) $\Delta V=0.0169$

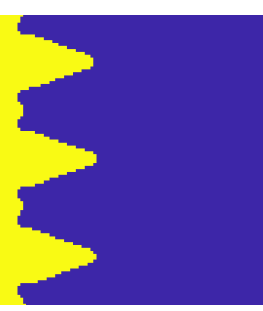

(b) $\Delta V=0.0386$

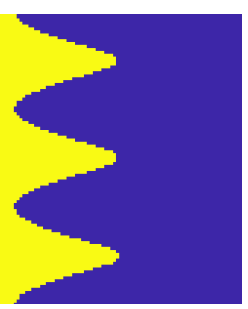

(c) $\Delta V=0.0483$

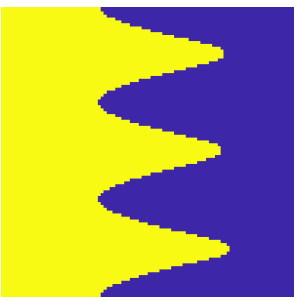

(d) $\Delta V=0.0579$

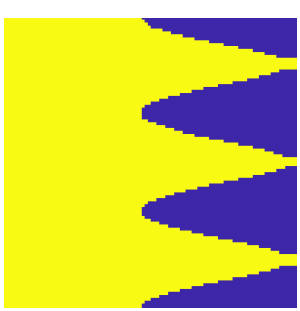

(e) $\Delta V=0.0700$

Figure 10: Design solutions solved for $f_{\mu}, h^{H C}=615, T^{C}=280, T^{H}=300$ and various electric potential differences, $\Delta V$. The plots illustrate that the design solutions are dependent on the electric potential difference and this model parameter should be taken into consideration in the design problem.

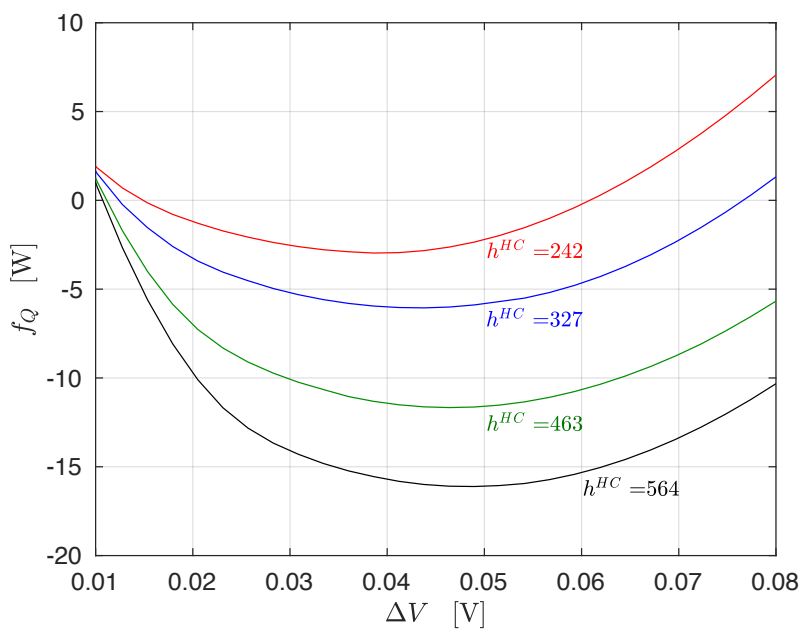

Figure 11: The relationships between $f_{Q}$ and $\Delta V$ for design solutions solved for $f_{Q}, T^{C}=280, T^{H}=300$ and $h^{H C}=\{242,327,463,564\}$. The plot shows that an increase of the convection coefficients results in an increase of the cooling power. The electric potential difference which results in the largest cooling power is increased as the convection coefficents are increased.

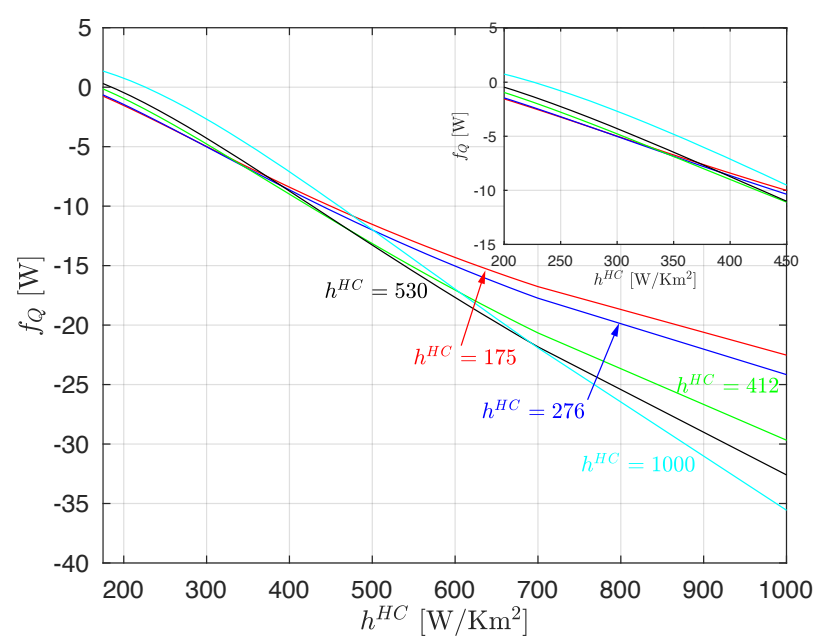

Figure 12: The relationships between $f_{Q}$ and $h^{H C}$ for the design solutions solved for $f_{Q}$ and $T^{C}=280, T^{H}=300$ and $h^{H C}=$ $\{175,276,412,530,1000\}$, where some of the design solutions are shown in Fig. 7. As the design solutions solved for one specific magnitude of $h^{H C}$ outperform the design solutions solved for different $h^{H C}$, we confidently conclude that we may attribute importance of the features of the design solutions with respect to $h^{H C}$. 
with the following interpolation functions [26]:

$$
\begin{aligned}
\alpha(x) & =\frac{(1-v) \alpha_{A} \sigma_{A}+v \alpha_{B} \sigma_{B}}{(1-v) \sigma_{A}+v \sigma_{B}} \\
\sigma(x) & =(1-v) \sigma_{A}+v \sigma_{B} \\
\kappa(x) & =(1-v) \kappa_{A}+v \kappa_{B}
\end{aligned}
$$

To demonstrate that this is a suitable approach, we have plotted the state fields along $x$ for the infinitely finely layered 2D design and infinitely finely layered 1D design in Fig. 15. With reference to the excellent fits of the state fields of the two modeling approaches, we confidently conclude that the two dimensional spike-shaped transitions of the design solutions actually account for a one dimensional feature. If the interpolation functions in Eqs. (10) are used to interpolate intermediate design variables, it is therefore sufficient to consider the equivalent one dimensional optimization problem, compare with Fig. 2.

The equivalence between the two dimensional infinitely finely layered and one dimensional infinitely layered design solutions is also observed for temperature dependent material parameters. These observations may be basis for the derivations of analytic optimization approaches in the future. However, it is important to clarify that the infinitely small feature of the design solutions in Fig. 15b may be challenging to manufacture and may cause large parasitic losses, for which reason designers most probably prefer to impose a minimum length scale as in the design solutions presented in this paper. If this is the case, the 1D and 2D models may deviate and it is therefore necessary to consider the full two dimensional optimization problem.

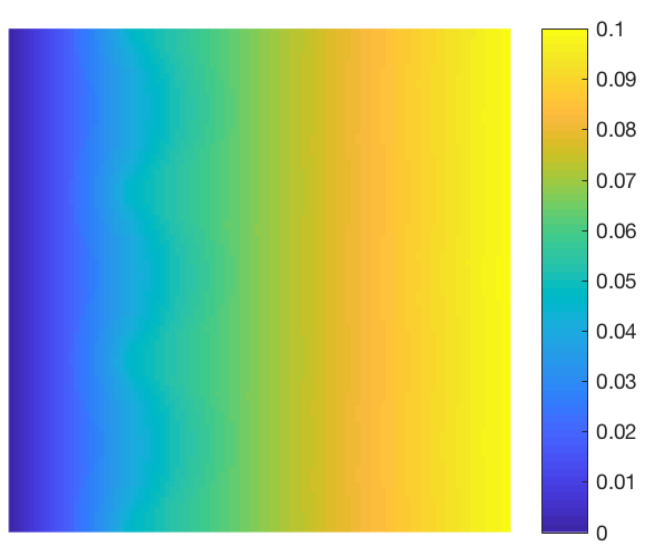

(a) Temperature state field

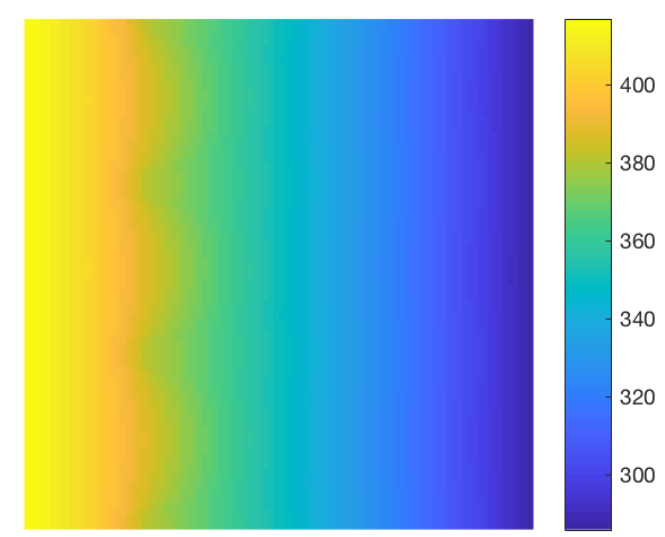

(b) Electric potential state field

Figure 13: The temperature and electric potential fields for the design solution solved for $f_{Q}, T^{C}=280, T^{H}=300$ and $h^{H C}=497$ in Fig. $7 \mathrm{~m}$. Despite the two dimensional features of the design solution, the state fields have relatively small gradients in the $y$-direction. 


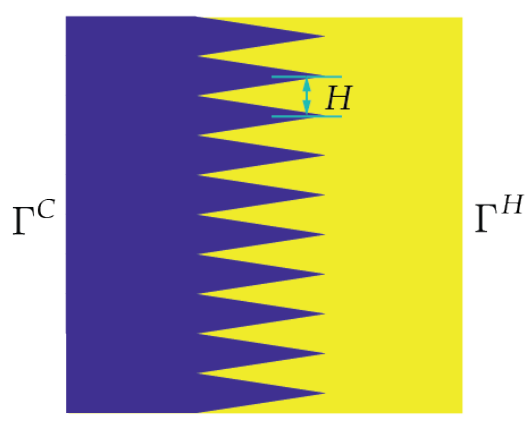

(a) Finitely layered 2D design

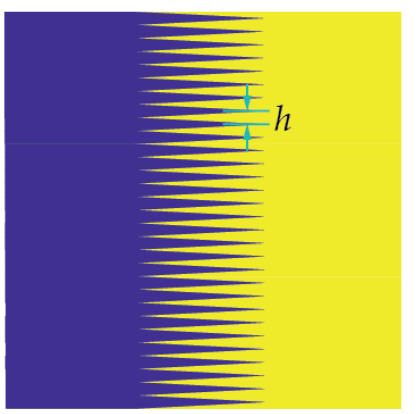

(b) Infinitely finely layered 2D design

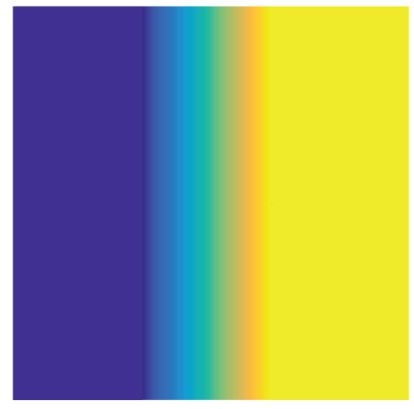

(c) Infinitely layered 1D design

Figure 14: Schematic of the steps in the decomposition of a Two Dimemsional (2D) finite layered design into an One Dimensional (1D) infite layered design. It is shown that the state fields of the design in (b) is equivalent to the state fields of the design in (c) if the interpolation functions in Eqs. (10) are used to interpolate intermediate design variables.

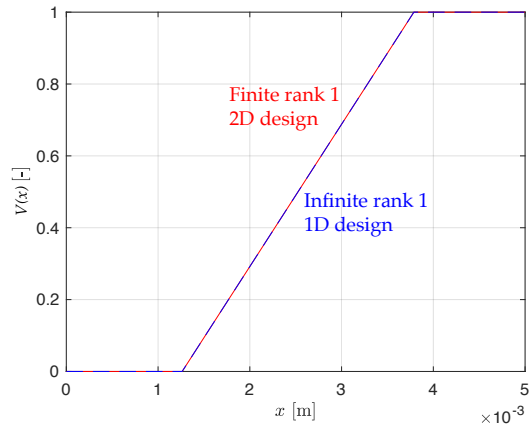

(a) The volume ratio between the materials

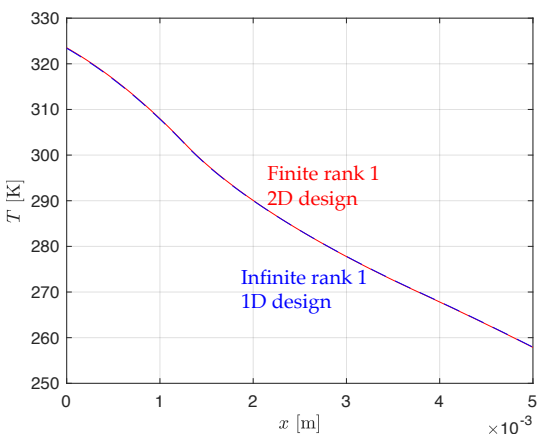

(b) The temperature field

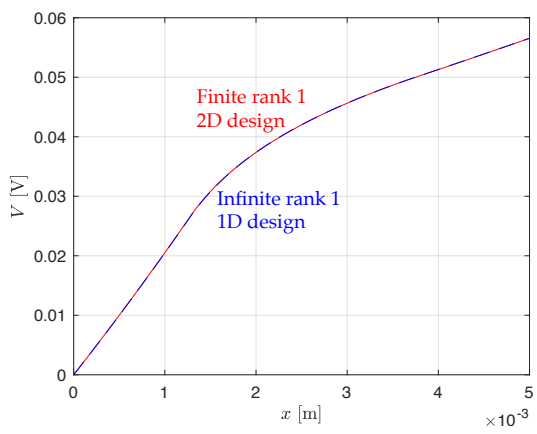

(c) The electric potential difference

Figure 15: Comparison between the state fields of the infinite layered two dimensional design and infinite layred one dimensional design in Figs. 14b and 14c. The two modeling approaches provide identical results for which reason it is concluded that the one dimensional optimization problem is adequate if the interpolation functions in Eq. (10) are used to interpolate intermediate design variables. 


\section{Discussion}

The topology optimization approach for thermoelectric coolers presented in this paper is related to wellaccepted work in the literature such as functionally graded materials [35], the compatibility approach [20], the thermoelectric homogenization approach [24, 26] and sizing approaches [36], however the methodology takes a completely different offset and modeling approach and therefore opens a complete new branch for optimization of thermoelectric coolers.

\subsection{Neglecting parasitic losses between material phases}

The parasitic losses between the material phases are neglected in the finite element modeling. This assumption is justified with reference to the work of Sakai, Kanno, Takahashi, Tamaki, Kusada, Yamada, and Abe [26], who manufactured and experimentally tested design solutions which consisted of two materials with a considerable amount of transitions between the material phases. These design solutions were manufactured and experimentally tested, and despite neglecting parasitic losses, good agreements between the analytic predictions and the experimentally tested and manufactured designs were shown. The parasitic losses could be included directly by formulating a more complex finite element model or indirectly through geometrical restrictions.

\subsection{The spike-shaped transitions between the material phases}

The design solutions can be characterized by three attributes: the volume ratio between the material phases, the length of the spike-shaped transition regions between the material phases, and the position of the transition between the material phases. The attributes of the design solutions are dependent on a large range of model parameters such as objective functions, convection coefficients, the temperature of the thermal reservoirs and the applied electric potential. To fully understand the interplays between the design solutions and the model parameters requires by an extensive parameter study with the numerical framework used in this study or an analytic optimization approach such as the one suggested but not fully developed in Sec. 3.5. Such study goes beyond the scope of the present paper.

\subsection{Temperature dependent materials}

The material parameters are assumed temperature independent, which is a non-physical assumption for most applications of thermoelectric energy conversion devices. It was decided to limit the modeling to temperature independent materials to simplify the interpretation of the spike-shaped design features. By using non-linear material parameters, it would be challenging to conclude whether the spike-shaped design features were occurring due to non-linear effects of the material parameters or to achieve intermediate effective material parameters of the stand-alone materials.

However, the topology optimization methodology can easily be extended to support temperature dependent material, see Lundgaard, Sigmund, and Bjørk [37] for more information.

\subsection{Manufacturability of the design solutions}

The level of geometrical complexity of the design solutions presented in this study is approximately similar to the design solutions manufactured and tested by Sakai and coworkers. With basis in this observation and with reference to advanced additive manufacturing methods [38], we assess that the design solutions are manufacturable with methodologies available today. Nevertheless, to manufacture and experimentally test the design solutions and hereby assess the difference between the numerical modeling and the experimental testing is a very important and interesting future study.

\section{Conclusion}

A density-based topology optimization approach is used to optimize the spatial distribution of two materials in order to optimize the performance of thermoelectric coolers. The design problems are solved for physically realistic boundary conditions and model dimensions, however, the design problems are purposely limited to temperature independent materials in order to ease the interpretation of the design features. The physical modeling is based on a fully coupled non-linear finite element model in two dimensions and steady state.

The most important findings are summarized in the following:

1. The topology optimization approach provides design solutions which outperform the classical segmentation approach with $48.7 \%$ and $11.4 \%$ for design problems solved for heat flux and coefficient of performance, respectively. We hereby conclude that topology optimization is a suited approach for optimizing thermoelectric coolers.

2. Design problems solved for average temperature and heat flux provide identical design solutions which is also concluded in the work of [10].

3. Design solutions solved for heat flux do not necessarily provide large coefficients of performances 
and design solutions solved for coefficients of performance do not necessarily provide large heat fluxes. The choice of objective function is therefore concluded to be a critical design parameter in design problems of thermoelectric coolers.

4. With basis in validation studies and cross-checks, it is shown that the applied electric potential difference, the temperatures of the thermal reservoirs and the convections coefficients are important model parameter and should be taken into consideration when optimizing thermoelectric coolers. Furthermore, we confidently conclude that the topology optimization approach is suited for taking these model parameters into account.

5. The design solutions are characterized by spikeshaped design features, which allow the designs solutions to operate locally in an intermediate state between the material phases. The two dimensional models can be decomposed into one dimensional models if interpolation functions of horizontally layered designs are used to define intermediate design variables. This may provide a road for developing new optimization approaches for thermoelectric coolers in the future.

The study provides new insight in the field of thermoelectric coolers and may provide guidance for future research aiming on developing high performing thermoelectric coolers.

\section{Acknowledgements}

The authors acknowledge the financial support received from the TopTen project sponsored by the Danish Council for Independent Research (DFF-4005-00320).

\section{References}

[1] David Michael Rowe. Thermoelectrics handbook: macro to nano. CRC press, 2005.

[2] Reiyu Chein and Guanming Huang. Thermoelectric cooler application in electronic cooling. Applied Thermal Engineering, 24 (14-15):2207-2217, 2004

[3] H Julian Goldsmid. Introduction to thermoelectricity, volume 121. Springer Science \& Business Media, 2009.

[4] Abraham Fedorovich Ioffe. Semiconductor thermoelements and thermoelectric cooling. 1957.

[5] J-P Fleurial, Alex Borshchevsky, Thierry Caillat, and Richard Ewell. New materials and devices for thermoelectric applications. In Energy Conversion Engineering Conference, 1997. IECEC97., Proceedings of the 32nd Intersociety, volume 2, pages 10801085. IEEE, 1997.

[6] Robert E Simons. Application of thermoelectric coolers for module cooling enhancement. Electronics Cooling, 6:18-25, 2000 .
[7] W. Seifert, E. Müller, and S. Walczak. Generalized analytic one-dimensional description of non-homogeneous TE cooler and generator elements based on the compatibility approach. International Conference on Thermoelectrics, ICT, Proceedings, pages 714-719, 2006. ISSN 1094-2734. doi: 10.1109/ICT.2006.331241.

[8] Zhixi Bian, Hongyun Wang, Qiaoer Zhou, and Ali Shakouri. Maximum cooling temperature and uniform efficiency criterion for inhomogeneous thermoelectric materials. Physical Review $B, 75: 245208,2007$. ISSN 10980121. doi: 10.1103/PhysRevB.75.245208.

[9] J Schilz, Eckhard Müller, L Helmers, YS Kang, Yasutoshi Noda, and Masayuki Niino. On the composition function of graded thermoelectric materials. In Materials science forum, volume 308, pages 647-652. Trans Tech Publ, 1999.

[10] Eckhard Müller, Steven Walczak, and Wolfgang Seifert. Optimization strategies for segmented Peltier coolers. Aerospace, 203(8):2128-2141, 2006. ISSN 18626300. doi: 10.1002/pssa.200521047.

[11] Zhixi Bian and Ali Shakouri. Cooling Enhancement Using Inhomogeneous Thermoelectric Materials. 2006 25th International Conference on Thermoelectrics, pages 6-9, 2006. ISSN 10942734. doi: 10.1109/ICT.2006.331365.

[12] Zhixi Bian and Ali Shakouri. Beating the maximum cooling limit with graded thermoelectric materials. Applied Physics Letters, 89(21):212101, 2006. ISSN 00036951. doi: 10.1063/1.2396895.

[13] Ole Sigmund. On the usefulness of non-gradient approaches in topology optimization. Structural and Multidisciplinary Optimization, 43(5):589-596, Mar 2011.

[14] York Christian Gerstenmaier and Gerhard Wachutka. Unified theory for inhomogeneous thermoelectric generators and coolers including multistage devices. Physical Review E - Statistical, Nonlinear, and Soft Matter Physics, 86:1-10, 2012. ISSN 15393755. doi: 10.1103/PhysRevE.86.056703.

[15] York Christian Gerstenmaier and Gerhard Wachutka. Rigorous theory of graded thermoelectric converters including finite heat transfer coefficients. Journal of Applied Physics, 122(20):204501, 2017.

[16] Martin Philip Bendsøe and Noboru Kikuchi. Generating optimal topologies in structural design using a homogenization method. Computer methods in applied mechanics and engineering, 71(2): 197-224, 1988.

[17] Martin Bendsøe and Ole Sigmund. Topology Optimization Theory, Methods and Applications. Springer, 2003.

[18] Ole Sigmund and Kurt Maute. Topology optimization approaches. Structural and Multidisciplinary Optimization, 48(6):1031-1055, 2013.

[19] Christian Lundgaard and Ole Sigmund. A density-based topology optimization methodology for thermoelectric energy conversion problems. Structural and Multidisciplinary Optimization, pages 1-16, 2018.

[20] T S Ursell and G J Snyder. Compatibility of segmented thermoelectric generators. Audio, Transactions of the IRE Professional Group on, pages 412-417, August 2002. ISSN 0-7803-7683-8.

[21] W. Seifert, E. Müller, and S. Walczak. Generalized analytic one-dimensional description of non-homogeneous TE cooler and generator elements based on the compatibility approach. International Conference on Thermoelectrics, ICT, Proceedings, pages 714-719, 2006. ISSN 1094-2734. doi: 10.1109/ICT.2006.331241.

[22] G. Jeffrey Snyder, Eric S. Toberer, Raghav Khanna, and Wolfgang Seifert. Improved thermoelectric cooling based on the Thomson effect. Physical Review B - Condensed Matter and Materials Physics, 86(4):1-8, 2012. ISSN 10980121. doi: 10.1103/PhysRevB.86.045202. 
[23] W Seifert, V Pluschke, and N F Hinsche. Thermoelectric cooler concepts and the limit for maximum cooling. Journal of physics. Condensed matter : an Institute of Physics journal, 255803 (25):255803, 2014. ISSN 1361-648X. doi: 10.1088/0953$8984 / 26 / 25 / 255803$

[24] Y Yang, S H Xie, F Y Ma, and C H Lei. On the effective thermoelectric properties of layered heterogeneous medium. Journal of Applied Physics, 111(1):3510, January 2012.

[25] Y Yang, F Y Ma, C H Lei, and Y Y Liu. Is thermoelectric conversion efficiency of a composite bounded by its constituents? Applied Physics Letters, 102(5):53905, January 2013.

[26] Akihiro Sakai, Tsutomu Kanno, Kouhei Takahashi, Hiromasa Tamaki, Hideo Kusada, Yuka Yamada, and Hiroya Abe. Breaking the trade-off between thermal and electrical conductivities in the thermoelectric material of an artificially tilted multilayer. Scientific reports, 4:6089, 2014.

[27] Chrisitan Lundgaard and Ole Sigmund. Design of segmented offdiagonal thermoelectric generators with topology optimization. Applied Energy, n/a(in review):n/a, 2018. doi: n/a.

[28] Elena E Antonova and David C Looman. Finite Elements for Thermoelectric Device Analysis in ANSYS. International Conference on Thermoelectrics, pages 1-4, aug 2000.

[29] Krister Svanberg. The method of moving asymptotesa new method for structural optimization. International journal for numerical methods in engineering, 24(2):359-373, 1987.

[30] Panagiotis Michaleris, Daniel A Tortorelli, and Creto A Vidal. Tangent operators and design sensitivity formulations for transient non-linear coupled problems with applications to elastoplasticity. International Journal for Numerical Methods in Engineering, 37(14):2471-2499, 1994.

[31] James K Guest and Jean H Prévost. Topology optimization of creeping fluid flows using a Darcy-Stokes finite element. International Journal for Numerical Methods in Engineering, 66(3): 461-484, 2006

[32] Ole Sigmund. Morphology-based black and white filters for topology optimization. Structural and Multidisciplinary Optimization, 33(4-5):401-424, jan 2007.

[33] SP Yushanov, LT Gritter, JS Crompton, and KC Koppenhoefer. Multiphysics analysis of thermoelectric phenomena. In Seventh Annual Conference on Multiphysics Modeling and Simulation. Proceedings of the 2011 COMSOL Conference, Boston, USA, 2011.

[34] Robert D. Cook, David S. Malkus, Michael E. Plesha, and Robert J. Witt. Concepts and applications of finite element analysis. John Wiley \& Sons, 4th edition, 2007.

[35] Wolfgang Seifert and Volker Pluschke. The extended concept of a self-compatible thermoelectric cooler. Physica Status Solidi (a), 211(4):917-923, 2014. ISSN 18626300. doi: 10.1002/pssa.201330392.

[36] Alexander Heghmanns and Michael Beitelschmidt. Parameter optimization of thermoelectric modules using a genetic algorithm. Applied Energy, 155:447-454, 2015.

[37] Chrisitan Lundgaard, Ole Sigmund, and Rasmus Bjørk. Topology optimization for segmented thermoelectric generators. $a b c, 1$ (123):123-123, 2018. ISSN 123. doi: 123.

[38] Bernhard Mueller. Additive manufacturing technologies-rapid prototyping to direct digital manufacturing. Assembly Automation, 32(2), 2012. 